$-\infty$

\section{NASA Contractor Report 191421}

ICASE Report No. 93-1
$1 N-34$

158533

P.31

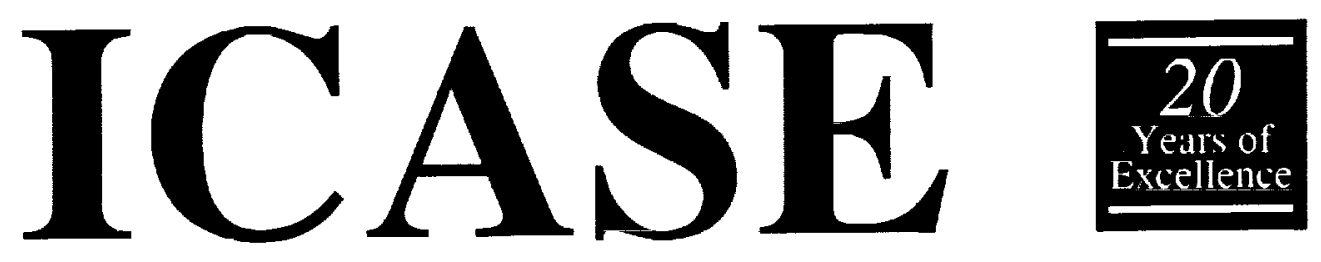

\title{
RENORMALIZATION GROUP ESTIMATES OF TRANSPORT \\ COEFFICIENTS IN THE ADVECTION OF A PASSIVE \\ SCALAR BY INCOMPRESSIBLE TURBULENCE
}

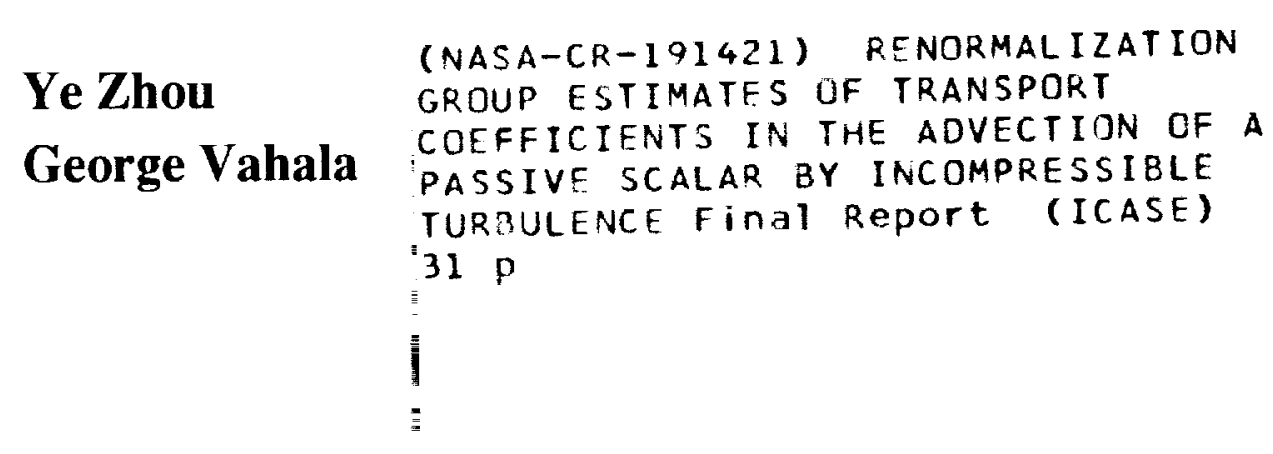

N93-23421

Unclas

$63 / 34 \quad 0158533$

NASA Contract No. NAS 1-19480

February 1993

Institute for Computer Applications in Science and Engineering

NASA Langley Research Center

Hampton, Virginia 23681-000I

Operated by the Universities Space Research Association

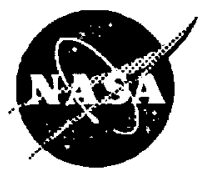

National Aeronautics and

Space Administration

Langley Research Center

Hampton, Virginia 23681-0001 


\section{ICASE Fluid Mechanics}

Due to increasing research being conducted at ICASE in the field of fluid mechanics, future ICASE reports in this area of research will be printed with a green cover. Applied and numerical mathematics reports will have the familiar blue cover, while computer science reports will have yellow covers. In all other aspects the reports will remain the same; in particular, they will continue to be submitted to the appropriate journals or conferences for formal publication. 
( 


\title{
Renormalization Group Estimates of Transport Coefficients in the Advection of a Passive Scalar by Incompressible Turbulence ${ }^{1}$
}

\author{
Ye Zhou \\ Institute for Computer Applications in Science and Engineering \\ NASA Langley Research Center, Hampton, VA 23681-0001 \\ George Vahala \\ Department of Physics, College of William and Mary \\ Williamsburg, VA 23185
}

\begin{abstract}
The advection of a passive scalar by incompressible turbulence is considered using recursive renormalization group procedures in the differential subgrid shell thickness limit. It is shown explicitly that the higher order nonlinearities induced by the recursive renormalization group procedure preserve Galilean invariance. Differential equations, valid for the entire resolvable wavenumber $k$ range, are determined for the eddy viscosity and eddy diffusivity coefficients and it is shown that higher order nonlinearities do not contribute as $k \rightarrow 0$, but have an essential role as $k \rightarrow k_{c}$, the cutoff wavenumber separating the resolvable scales from the subgrid scales. The recursive renormalization transport coefficients and the associated eddy Prandtl number are in good agreement with the $k$-dependent transport coefficients derived from closure theories and experiments.
\end{abstract}

\footnotetext{
${ }^{1}$ This research was supported by the National Aeronautics and Space Administration under NASA Contract No. NAS1-19480 while the authors were in residence at the Institute for Computer Applications in Science and Engineering (ICASE), NASA Langley Research Center, Hampton, VA 23681-0001.
} 


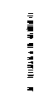

$\underline{\underline{i}}$

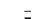




\section{Introduction}

The turbulent transport of a passive scalar, while serving sound pedagogical purposes, is also of interest in the spreading of temperature, humidity and pollution in the atmosphere as well as in other problems (Csanady ${ }^{1}$ ). Here we shall apply recursive renormalization group (RNG) procedures to the subgrid modeling of a passive scalar field $T(\mathbf{k}, t)$ being advected by a turbulent Navier-Stokes velocity field $\mathbf{u}(\mathbf{k}, t)$. Subgrid modeling is necessary for the high-Reynolds number turbulent flows of interest because of the limitations of current and foreseeable supercomputers ${ }^{2}$. Another advantage of considering the problem of passive scalar transport is that the spectral transport coefficients (eddy diffusivity and eddy viscosity) determined from our RNG theory can be compared to those arising from closure-based theories ${ }^{3,4}$. It should be noted that the transport coefficients in these closure theories are determined over the whole resolvable scales.

Recently, two distinct approaches of RNG to fluid turbulence have arisen: one based on the work of Forster et. al. ${ }^{5}$, called $\epsilon$-RNG, and the other based on Rose ${ }^{6}$, called recursiveRNG. Some aspects of these two approaches have been discussed ${ }^{7}$. In particular, we point out here that in $\epsilon$-RNG, a small parameter $\epsilon$ is introduced through the forcing correlation function. Yakhot \& Orszag $^{8}$ have extrapolated $\epsilon \ll 1$ to $\epsilon \rightarrow 4$ in order to reproduce the Kolmogorov energy spectrum. Furthermore, it is also necessary to take the distant interaction limit ${ }^{9}, k \rightarrow 0$. Thus, it is difficult to compare the transport coefficients generated by Kraichnan ${ }^{3}$ and Chollet $^{4}$, with that determined from $\epsilon$-RNG.

In this paper, we continue our application of recursive $\mathrm{RNG}^{10-11}$ to turbulence. The basic differences between the two RNG procedures are that in recursive RNG:

(i) The $\epsilon$-expansion is not applied.

(ii) The turbulent transport coefficients are determined for the whole resolvable wavenumber scales,

(iii) Higher order nonlinearities are generated in the renormalized momentum equation.

In Sec. 2 we derive the renormalized evolution equations for the passive scalar $T(\mathrm{k}, t)$ and the fluid velocity $\mathbf{u}(\mathbf{k}, t)$ as well as the recursion relations from which the eddy diffusivity and eddy viscosity can be determined. Because of the presence of higher order nonlinearities in the renormalized equations, it is not apparent that Galilean invariance is still preserved. These questions are addressed in Sec. 3 where we prove that the RNG-evolution equations are Galilean invariant: a property deemed necessary in any subgrid mode ${ }^{12}$. It has been found very difficult to find fixed points for the RNG-difference recursion relations if the subgrid 
shell thickness is chosen too small ${ }^{10.11}$. If recursion RNG procedures are to be employed successfully in more complicated flow problems, then it is necessary that these difference recursion relations be simplified. In Sec. 4 we proceed to the differential limit of these recursion relations, paying careful attention to the $k \rightarrow 0$ limit. We show that the higher order RNG-induced nonlinearities do not contribute to the $k \rightarrow 0$ limit of the RNG recursion relations, but play a significant role for $k \rightarrow k_{c}$, where $k_{c}$ is the wavenumber that separates the resolvable scale from the subgrid scale. In the Appendix, we contrast our ordinary differential equations for the RNG eddy viscosity and diffusivity with that generated by Yakhot-Orszag ${ }^{8}$ by their $\epsilon$-RNG, a theory that is valid only in the distant interaction limit $k \rightarrow 0$. In contrast, in the recursive RNG approach, the differential equations for the eddy transport coefficients are valid over the whole resolvable scales and not just at $k \rightarrow 0$, and no $\epsilon$ expansion is needed. The turbulent transport coefficients for the second moments (i.e., for the time evolution of $U_{\alpha \beta}(\mathbf{k}, t)=<u_{\alpha}(\mathbf{k}, t) u_{\beta}(-\mathbf{k}, t)>$ and the scalar variance $\left.\Theta(\mathbf{k}, t)=<T(\mathbf{k}, t) T(-\mathbf{k}, t)>\right)$ are determined in Sec. 5. In particular, the importance of the RNG-induced higher order nonlinearities is very apparent. The spectral eddy viscosity, diffusivity and Prandtl number are derived in Sec. 6, while in Sec. 7 we present our conclusions.

\section{Renormalized momentum equation for velocity and passive scalar}

We consider a passive scalar $T(\mathrm{k}, t)$ being advected by incompressible turbulence

$$
\left[\frac{\partial}{\partial t}+\mu_{0} k^{2}\right] T(\mathbf{k}, t)=-i k_{\alpha} \int d^{3} j u_{\alpha}(\mathbf{k}-\mathbf{j}, t) T(\mathbf{j}, t)
$$

with the turbulent velocity field $\mathbf{u}(\mathbf{k}, t)$ being determined from the Navier-Stokes equation

$$
\left[\frac{\partial}{\partial t}+\nu_{0} k^{2}\right] u_{\alpha}(\mathbf{k}, t)=M_{\alpha \beta \gamma}(k) \int d^{3} j u_{\beta}(\mathbf{j}, t) u_{\gamma}(\mathbf{k}-\mathbf{j}, t)+f_{\alpha}(\mathbf{k}, t)
$$

Summation over repeated subscripts is understood, and

$$
M_{\alpha \beta \gamma}(k)=k_{\beta} D_{\alpha \gamma}(k)+k_{\gamma} D_{\alpha \beta}(k), \quad \text { and } \quad D_{\alpha \beta}(k)=\delta_{\alpha \beta}-k_{\alpha} k_{\beta} / k^{2}
$$

Here $\mu_{0}$ is the molecular diffusivity, $\nu_{0}$ the molecular viscosity and $f_{\alpha}$ a random forcing term. The forcing correlation is given by

$$
<f_{\alpha}(k, t) f_{\beta}\left(k^{\prime}, t^{\prime}\right)>=D_{0} k^{-y} D_{\alpha \beta}(k) \delta\left(k+k^{\prime}\right) \delta\left(t-t^{\prime}\right)
$$

where $D_{0}$ denotes the intensity of the forcing, and $y$ is an appropriately chosen exponent so as to recover the Kolmogorov energy scaling in the inertial range $(y=3)$. 


\subsection{An outline of the recursive RNG procedure}

Since the details of the recursive RNG procedure for Navier-Stokes turbulence have been presented before ${ }^{6,10-11}$, we just briefly outline the steps here:

(i) The subgrid wavenumber region $\left(k_{c}, k_{d}\right)$ is partitioned into $N$-shells

$$
k_{c} \equiv k_{N}<k_{N-1}<\ldots<k_{1}<k_{0} \equiv k_{d}
$$

where $k_{c}$ is the wavenumber separating the resolvable from the subgrid scales and $k_{d}$ is the Kolmogorov dissipation wavenumber. $k_{n}=f^{n} k_{0}, n=0, \ldots, N$, where $f$ is a factor, $0<f<1$, measuring the coarseness of the subgrid partitioning. The limit $f \rightarrow 1$ corresponding to a differential partitioning of the subgrid region $(N \rightarrow \infty)$.

(ii) The subgrid modes for the first shell, $k_{1}<k \leq k_{0}$, are eliminated from the resolvable scale equation by the solution of the subgrid scale equation.

(iii) A subgrid scale average is performed over the resultant resolvable scale equation. This will result not only in the introduction of the subgrid scale energy (or equivalently, forcing) spectrum, but it also results in a new triple nonlinearity and nonlocal eddy damping function in the resolvable momentum equation $\left(k \leq k_{c}\right)$.

(iv) The above steps are repeated for each successive subgrid shell until all the subgrid scales have been removed.

(v) Since the subgrid scales evolve on a faster time scale than the resolvable scales, a multiple time-scale analysis can be performed to simplify the eddy damping function. The resultant eddy viscosity is a fixed point of an integro-difference recursion relation.

(vi) The recursion relation for the eddy viscosity and the renormalized Navier- Stokes equation are rescaled.

It should be emphasized that there are two singular limits ${ }^{7}: f \rightarrow 1$ and $k \rightarrow 0$. A careful analysis must be done regarding these two limits and the associated averaging operations. We will address this issue here in the present paper.

\subsection{An asymmetry in the renormalized passive scalar equation}

The details of the implementation of the recursive RNG procedure to the advection of a passive scalar is a straightforward generalization of that for Navier-Stokes turbulence (see e.g. Hossain ${ }^{13}$ ) and so will not be presented here. Here we comment on a symmetrization 
procedure that is standard when dealing with Navicr-Stokes turbulence, but which can not be applied to the passive scalar problem.

Consider the removal of the first subgrid shell and use the usual notation

$$
u_{\alpha}(\mathbf{k}, t)= \begin{cases}u_{\alpha}^{>}(\mathrm{k}, t) & \text { if } k_{1}<k<k_{0} \\ u_{\alpha}^{<}(\mathrm{k}, t) & \text { if } k<k_{1}\end{cases}
$$

and

$$
T(\mathbf{k}, t)=\left\{\begin{array}{ll}
T^{>}(\mathbf{k}, t) & \text { if } k_{1}<k<k_{0} ; \\
T^{<}(\mathbf{k}, t) & \text { if } k<k_{1}
\end{array},\right.
$$

We find for $k<k_{1}$, the resolvable scale passive scalar and Navier-Stokes equation can be written as

$$
\begin{aligned}
{\left[\frac{\partial}{\partial t}+\mu_{0} k^{2}\right] T^{<}(\mathbf{k}, t)=-k_{\alpha} \int } & d^{3} j\left[u_{\alpha}^{<}(\mathbf{k}-\mathbf{j}, t) T^{<}(\mathbf{j}, t)+u_{\alpha}^{>}(\mathrm{k}-\mathbf{j}, t) T^{<}(\mathbf{j}, t)\right. \\
& \left.+u_{\alpha}^{<}(\mathbf{k}-\mathbf{j}, t) T^{>}(\mathbf{j}, t)+u_{\alpha}^{>}(\mathbf{k}-\mathbf{j}, t) T^{>}(\mathbf{j}, t)\right]
\end{aligned}
$$

and

$$
\begin{aligned}
{\left[\frac{\partial}{\partial t}+\nu_{0} k^{2}\right] u_{\alpha}^{<}(\mathbf{k}, t)=} & f_{\alpha}^{<}(\mathbf{k}, t)+M_{\alpha \beta \gamma}(k) \int d^{3} j\left[u_{\beta}^{<}(\mathbf{j}, t) u_{\gamma}^{<}(\mathbf{k}-\mathbf{j}, t)\right. \\
& \left.+2 u_{\beta}^{>}(\mathbf{j}, t) u_{\gamma}^{<}(\mathbf{k}-\mathbf{j}, t)+u_{\beta}^{>}(\mathbf{j}, t) u_{\gamma}^{>}(\mathbf{k}-\mathbf{j}, t)\right] .
\end{aligned}
$$

The factor 2 in the Navier-Stokes Eq. (9) arises from the symmetry in the $\mathbf{j} \leftrightarrow \mathbf{k}-\mathbf{j}$ interchange.

We assume isotropy for both the velocity field and passive scalar, so that the subgrid velocity - passive scalar correlations are zero (Lesieur ${ }^{14}$ ):

$$
<u^{>} T^{>}>=0
$$

where $\langle\ldots\rangle$ represents averaging over the subgrid scales.

On applying the recursive RNG procedure to eliminate the subgrid fields in Eqs. (8) and (9), the second term on the RHS of Eq. (8) requires special attention. In Ref. 6, the frozen flow velocity field is prescribed, so that this term plays no role in the renormalization procedure. However, here we are considering a passive scalar field being advected by a turbulent velocity field which is itself determined from Navier-Stokes turbulence. As a result, this term must be treated on the same footing as the others. For this second term on the RHS of Eq. (8) we have the wavenumber restrictions, $j, k<k_{1}$ while $k_{1}<|\mathbf{k}-\mathbf{j}|<$ $k_{0}$ is in the subgrid scale. This can only be achieved when $k$ and $j$ are located near $k_{1}$. Thus $u_{\alpha}^{>}(\mathbf{k}-\mathbf{j}, t) T^{<}(\mathbf{j}, t)$ with the wavenumber restriction discussed above is different from $u_{\alpha}^{>}(\mathbf{j}, t) T^{<}(\mathbf{k}-\mathbf{j}, t)$ with $k,|\mathbf{k}-\mathbf{j}|<k_{1}$ and $k_{1}<j<k_{0}$. Hence one can not interchange the variables $\mathbf{j}$ and $\mathbf{k}-\mathbf{j}$ by standard symmetry arguments as done by Hossain ${ }^{13}$. 


\subsection{The renormalized Navier-Stokes and passive scalar equations}

After removing the $n^{\text {th }}$ subgrid shell, the renormalized passive scalar equation takes the form

$$
\begin{array}{r}
{\left[\frac{\partial}{\partial t}+\mu_{n}(k) k^{2}\right] T(\mathbf{k}, t)=-i k_{\alpha} \int d^{3} j u_{\alpha}^{<}(\mathbf{k}-\mathbf{j}, t) T^{<}(\mathbf{k}, t)} \\
-k_{\alpha} \sum_{h=1}^{n} \int d^{3} j d^{3} j^{\prime} \frac{j \beta}{\mu_{n-h}(j) j^{2}} u_{\alpha}^{<}(\mathbf{k}-\mathbf{j}, t) u_{\beta}^{<}\left(\mathbf{j}-\mathbf{j}^{\prime}, t\right) T^{<}\left(\mathbf{j}^{\prime}, t\right) \\
-i k_{\alpha} \sum_{h=1}^{n} \int d^{3} j d^{3} j^{\prime} \frac{M_{\alpha \beta \gamma}(\mathbf{k}-\mathbf{j})}{\nu_{n-h}(|\mathbf{k}-\mathbf{j}|)|\mathbf{k}-\mathbf{j}|^{2}} u_{\beta}^{<}\left(\mathbf{j}^{\prime}, t\right) u_{\gamma}^{<}\left(\mathbf{k}-\mathbf{j}-\mathbf{j}^{\prime}, t\right) T^{<}(\mathbf{j}, t)
\end{array}
$$

where the last term on the RHS differs from that of Hossain ${ }^{13}$.

The restriction on the wavenumbers are the following:

$$
\begin{aligned}
& k_{N}<j<k_{N-1} \text { in the second integral } \\
& k_{N}<|\mathbf{k}-\mathbf{j}|<k_{N-1} \text { in the third integral. }
\end{aligned}
$$

The other wavenumber constraints are as indicated by the superscript on the fields $\mathbf{u}$ and $T$.

The renormalized Navier-Stokes equation has the following form ${ }^{11}$ :

$$
\begin{array}{r}
{\left[\partial / \partial t+\nu_{n}(k) k^{2}\right] u_{\alpha}(\mathbf{k}, t)=f_{\alpha}(\mathbf{k}, t)+M_{\alpha \beta \gamma}(\mathbf{k}) \int d^{3} j u_{\beta}^{<}(\mathbf{j}, t) u_{\gamma}^{<}(\mathbf{k}-\mathbf{j}, t)} \\
+2 M_{\alpha \beta \gamma}(\mathbf{k}) \sum_{h=1}^{n} \int d^{3} j d^{3} j^{\prime} \frac{1}{\nu_{n-h}(j) j^{2}} M_{\beta \beta^{\prime} \gamma^{\prime}}(j) u_{\beta^{\prime}}^{<}\left(\mathbf{j}^{\prime}, t\right) u_{\gamma^{\prime}}^{<}\left(\mathbf{j}-\mathbf{j}^{\prime}, t\right) u_{\gamma}^{<}(\mathbf{k}-\mathbf{j}, t)
\end{array}
$$

where $j$ is restricted to the subgrid shell in the second integral. Again, all other wavenumber constraints are as indicated by the superscript.

\subsection{Recursion relations for eddy viscosity and diffusivity}

Although the second term on the RHS of Eq. (8) contributes a new triple nonlinear term, it does not make any contribution to the renormalized eddy diffusivity in the momentum equation in the process of removing the next subgrid shell. The reason is the following:

$$
2^{\text {nd }} \quad \operatorname{term} \sim M_{\alpha \beta \gamma}(\mathbf{k}-\mathbf{j})<u_{\beta}^{>}\left(\mathbf{j}^{\prime}, t\right) u_{\gamma}^{>}\left(\mathbf{k}-\mathbf{j}-\mathbf{j}^{\prime}, t\right)>T^{<}(\mathbf{j}, t) \rightarrow 0
$$

since the ensemble average will generate a delta function $\delta(\mathbf{k}-\mathbf{j})$ while $\mathbf{k}-\mathbf{j}$ is in the subgrid range. This is impossible, and so this second term can not contribute to the eddy diffusivity.

After the removal of the $(n+1)^{t h}$ subgrid shell, the spectral eddy viscosity in the renormalized momentum equation is determined by the recursion relation ${ }^{11}$ 


$$
\nu_{n+1}(k)=\nu_{n}(k)+\delta \nu_{n}(k)
$$

where

$$
\delta \nu_{n}(k)=\frac{D_{0}}{k^{2}} \sum_{h=0}^{n} \int d^{3} j \frac{L(k, j, q)|\mathbf{k}-\mathbf{j}|^{-y}}{\nu_{h}(j) j^{2} \nu(|\mathbf{k}-\mathbf{j}|)|\mathbf{k}-\mathbf{j}|^{2}}
$$

and

$$
L(k, j, q)=-\frac{k j\left(1-z^{2}\right)\left[z q^{2}-k j\right]}{q^{2}}
$$

with $\mathbf{k} \cdot \mathbf{j}=k j z$. This difference equation, after rescaling, has been solved by Zhou et al. ${ }^{11}$ and fixed points were readily determined for finite $f \leq 0.7$. However, it was very difficult to determine fixed points for finer subgrid partition factor $f>0.7$. In Sec. 4 we shall pass to the differential subgrid limit $f \rightarrow 1$ and determine an ordinary differential equation (o.d.e) for the renormalized eddy viscosity over the entire resolvable scale which can be readily integrated.

In a similar fashion, the spectral eddy diffusivity in the renormalized passive scalar equation can be shown to be given after the removal of the $(n+1)^{t h}$ subgrid shell, by

$$
\mu_{n+1}(k)=\mu_{n}(k)+\delta \mu_{n}(k)
$$

where

$$
\delta \mu_{n}(k)=\frac{k_{\alpha} k_{\beta}}{k^{2}} \sum_{h=0}^{n} \int d^{3} j \frac{D_{\alpha \beta}(k-j) Q(|\mathbf{k}-\mathbf{j}|)}{\mu_{n-h}(j) j^{2}}
$$

The renormalized eddy viscosity and diffusivity are defined as the fixed point of these recursion relations.

\subsection{Rescaling of the recursion relation and momentum equations}

From the self-similarity properties of the forcing and energy spectrum in the subgrid range, we expect that the viscosity $\nu_{n+1}$ to be simply related to $\nu_{n}$ for large $n$, while the diffusivity $\mu_{n+1}$ is simply related to $\mu_{n}$. A rescaling can be performed on the recursion relation. In particular, consider

$$
k \rightarrow k_{n+1} \tilde{k}
$$

and define

$$
\tilde{\nu}_{n}(\tilde{k}) \equiv k_{n+1}^{(y+1) / 3} \nu_{n}\left(k_{n+1} \tilde{k}\right) \quad \text { for } \quad \tilde{k} \leq 1
$$


Unless mentioned otherwise, we drop the tilde notation on the wavenumber, and note that $0<k \leq 1$. The recursion relation for the eddy viscosity becomes

$$
\tilde{\nu}_{n+1}(k)=f^{(y+1) / 3}\left[\tilde{\nu}_{n}(f k)+\delta \tilde{\nu}_{n}(f k)\right]
$$

where

$$
\begin{gathered}
\delta \tilde{\nu}_{n}(f k)=f^{-(y+1)}\left[\frac{D_{0}}{k^{2}} \int d^{3} j \frac{L(k, j, q)|\mathbf{k}-\mathbf{j}|^{-y}}{\tilde{\nu}_{n}(f j) j^{2} \tilde{\nu}_{n}(f|\mathbf{k}-\mathbf{j}|)|\mathbf{k}-\mathbf{j}|^{2}}\right. \\
\left.+\sum_{h=1}^{n} \frac{D_{0}}{k^{2}} \int d^{3} j f^{-h(y+1) / 3} \frac{L(k, j, q)|\mathbf{k}-\mathbf{j}|^{-y}}{\tilde{\nu}_{n-h}\left(f^{h+1} j\right) j^{2} \tilde{\nu}_{n}(f|\mathbf{k}-\mathbf{j}|)|\mathbf{k}-\mathbf{j}|^{2}}\right],
\end{gathered}
$$

and the summation term arises from the triple nonlinearity induced by the recursive RNG procedure. The recursion relation for the eddy diffusivity is

$$
\tilde{\mu}_{n+1}(k)=f^{(m+1) / 2}\left[\tilde{\mu}_{n}(f k)+\delta \tilde{\mu}_{n}(f k)\right]
$$

with

$$
\begin{array}{r}
\delta \tilde{\mu}_{n}(f k)=\frac{f^{-(m+1)}}{4 \pi}\left[\frac{k_{\alpha} k_{\beta}}{k^{2}} \int d^{3} j \frac{D_{\alpha \beta}(k-j)|\mathbf{k}-\mathbf{j}|^{-(m+2)}}{\tilde{\mu}_{n}(f j) j^{2}}\right. \\
\left.+\sum_{h=1}^{n} \frac{k_{\alpha} k_{\beta}}{k^{2}} f^{-h(m+1) / 2} \int d^{3} j \frac{D_{\alpha \beta}(k-j)|\mathbf{k}-\mathbf{j}|^{-(m+2)}}{\tilde{\mu}_{n-h}\left(f^{h+1} j\right) j^{2}}\right]
\end{array}
$$

where again the second term on RHS of Eq. (24) arises from the induced triple nonlinearities. We have introduced the parameter $m=5 / 3$. These equations are valid for any $k$ in the resolvable scales: $0<k \leq k_{c}$. In the limit $k \rightarrow 0$, the triple term contribution $\rightarrow 0$, as will be shown in Sec. 4.1.

The final renormalized passive scalar equation is

$$
\begin{array}{r}
{\left[\frac{\partial}{\partial t}+\mu(k) k^{2}\right] T(\mathbf{k}, t)=-i k_{\alpha} \int d^{3} j u_{\alpha}^{<}(\mathbf{k}-\mathbf{j}, t) T^{<}(\mathbf{k}, t)} \\
-\frac{k_{\alpha}}{\mu\left(k_{c}\right) k_{c}^{\frac{m+1}{2}}} \int d^{3} j d^{3} j^{\prime} \frac{j_{\beta}}{j^{\frac{3-m}{2}}} u_{\alpha}^{<}(\mathbf{k}-\mathbf{j}, t) u_{\beta}^{<}\left(\mathbf{j}-\mathbf{j}^{\prime}, t\right) T^{<}\left(\mathbf{j}^{\prime}, t\right) \\
-\frac{i k_{\alpha}}{\nu\left(k_{c}\right) k_{c}^{\frac{m+1}{2}}} \int d^{3} j d^{3} j^{\prime} \frac{M_{\alpha \beta \gamma}(\mathbf{k}-\mathbf{j})}{|\mathbf{k}-\mathbf{j}|^{\frac{3-m}{2}}} u_{\beta}^{<}\left(\mathbf{j}^{\prime}, t\right) u_{\gamma}^{<}\left(\mathbf{k}-\mathbf{j}-\mathbf{j}^{\prime}, t\right) T^{<}(\mathbf{j}, t)
\end{array}
$$

while the final renormalized Navier-Stokes equation is

$$
\begin{array}{r}
{\left[\partial / \partial t+\nu(k) k^{2}\right] u_{\alpha}(\mathbf{k}, t)=f_{\alpha}(\mathbf{k}, t)+M_{\alpha \beta \gamma}(\mathbf{k}) \int d^{3} j u_{\beta}^{<}(\mathbf{j}, t) u_{\gamma}^{<}(\mathbf{k}-\mathbf{j}, t)} \\
+2 \frac{1}{\nu\left(k_{c}\right) k_{c}^{\frac{y+1}{3}}} M_{\alpha \beta \gamma}(\mathbf{k}) \int d^{3} j d^{3} j^{\prime} \frac{1}{j^{\frac{5-y}{3}}} M_{\beta \beta^{\prime} \gamma^{\prime}}(j) u_{\beta^{\prime}}^{<}\left(\mathbf{j}^{\prime}, t\right) u_{\gamma^{\prime}}^{<}\left(\mathbf{j}-\mathbf{j}^{\prime}, t\right) u_{\gamma}^{<}(\mathbf{k}-\mathbf{j}, t) .
\end{array}
$$




\section{Galilean invariance of the renormalized Navier-Stokes and passive scalar equations}

Before we proceed further, we turn our attention to the question of the Galilean invariance of the renormalized Navier-Stokes and passive scalar equations (25) and (26). The importance of Galilean invariance in turbulence modelling has been emphasized by Speziale ${ }^{12}$. To be consistent with the basic physics, it is required that the description of the turbulence be the same in all inertial frames of reference. The appearance of the triple nonlinear term, which is a function of the resolvable scales velocity fields, makes the property of the Galilean invariance of our recursive RNG procedure not transparent. We now show that both the renormalized Navier-Stokes equation and the renormalized passive scalar equation are Galilean invariant.

\subsection{Galilean invariance in Navier-Stokes equation: A review}

The Galilean transformation is

$$
\mathbf{x} \rightarrow \mathbf{x}^{*}-\mathbf{U}_{0} t^{*} \quad t \rightarrow t^{*}
$$

Thus, one has

$$
\mathbf{u}=\mathbf{u}^{*}-\mathbf{U}_{0}, \quad \frac{\partial}{\partial x}=\frac{\partial}{\partial x^{*}} \quad \frac{\partial}{\partial t}=\frac{\partial}{\partial t^{*}}+\mathbf{U}_{0} \cdot \frac{\partial}{\partial x^{*}} .
$$

While the Galilean transformation for the Navier-Stokes equation in physical space is trivial, the Galilean transformation in wavenumber space is less obvious, due to the lack of differential operations. For convenience, we first review how Galilean invariance is preserved for the Navier-Stokes equation in the wavenumber space.

Under the Galilean transformation, the LHS of the Navier-Stokes equation [cf. Eq. (2)] becomes

$$
\begin{array}{r}
\frac{\partial u_{\alpha}^{*}\left(\mathbf{k}^{*}, t\right)}{\partial t^{*}}+U_{0 \beta} i k_{\beta}^{*} u_{\alpha}^{*}\left(\mathbf{k}^{*}, t\right)+\nu_{0} k^{* 2}\left[-U_{0 \alpha} \delta\left(\mathbf{k}^{*}\right)+u_{\alpha}^{*}\left(\mathbf{k}^{*}, t\right)\right] \\
=\frac{\partial u_{\alpha}^{*}\left(\mathbf{k}^{*}, t\right)}{\partial t^{*}}+U_{0 \beta} i k_{\beta}^{*} u_{\alpha}^{*}\left(\mathbf{k}^{*}, t\right)+\nu_{0} k^{* 2} u_{\alpha}^{*}\left(\mathbf{k}^{*}, t\right)
\end{array}
$$

where in the last step, we have used the the $\delta$ function property $k^{* 2} \delta\left(\mathbf{k}^{*}\right)=0$.

Under the Galilean transformation, the RHS of the Navier-Stokes equation [c.f. Eq. 2] becomes 


$$
\begin{array}{r}
M_{\alpha \beta \gamma}\left(k^{*}\right) \int d^{3} j\left[u_{\beta}^{*}\left(\mathbf{j}^{*}, t\right)-U_{0 \beta} \delta\left(\mathbf{j}^{*}\right)\right]\left[u_{\beta}^{*}\left(\mathbf{k}^{*}-\mathbf{j}^{*}, t\right)-U_{0 \gamma} \delta\left(\mathbf{k}^{*}-\mathbf{j}^{*}\right)\right] \\
=M_{\alpha \beta \gamma}\left(k^{*}\right) \int d^{3} j^{*} u_{\beta}^{*}\left(\mathbf{j}^{*}, t\right) u_{\gamma}^{*}\left(\mathbf{k}^{*}-\mathbf{j}^{*}, t\right)+i U_{0 \beta} k_{\beta}^{*} u_{\alpha}^{*}\left(\mathbf{k}^{*}, t\right)
\end{array}
$$

where we have used the property of the $\delta$ function, the incompressible condition, and

$$
M_{\alpha \beta \gamma}\left(k^{*}\right) U_{0 \beta} u_{\gamma}^{*}\left(\mathbf{k}^{*}, t\right)=U_{0 \beta} k_{\beta}^{*} u_{\alpha}^{*}\left(\mathbf{k}^{*}, t\right) /(2 i)
$$

Thus, as expected, the Navier-Stokes equation is invariant under a Galilean transformation due to the cancellation of the second term on the RHS of Eqs. (29)-(30).

\subsection{The renormalized Navier-Stokes equation under a Galilean transformation}

To show that the renormalized Navier-Stokes equation is invariant under a Galilean transformation, we need only consider the recursive RNG induced triple nonlinear term, denoted by $N S_{T}$ :

$$
N S_{T} \equiv 2 M_{\alpha \beta \gamma}(k) \int d^{3} j d^{3} j^{\prime}\left(\frac{j}{k_{c}}\right)^{4 / 3} \frac{M_{\beta \beta^{\prime} \gamma^{\prime}}(j)}{\nu\left(k_{c}\right) j^{2}} u_{\beta^{\prime}}\left(\mathbf{j}-\mathbf{j}^{\prime}, t\right) u_{\gamma^{\prime}}\left(\mathbf{j}^{\prime}, t\right) u_{\gamma}(\mathbf{k}-\mathbf{j}, t)
$$

It is important to note that $j$ is in the subgrid.

Under a Galilean transformation, Eq. (32) becomes

$$
\begin{gathered}
N S_{T}^{*}=2 M_{\alpha \beta \gamma}\left(k^{*}\right) \int d^{3} j^{*} d^{3} j^{\prime *}\left(\frac{j^{*}}{k_{c}}\right)^{4 / 3} \frac{M_{\alpha \beta \gamma}\left(j^{*}\right)}{\nu\left(k_{c}^{*}\right) j^{* 2}}\left[u_{\beta^{\prime}}^{*}\left(\mathbf{j}^{*}-\mathbf{j}^{\prime *}, t\right)-U_{0 \beta^{\prime}} \delta\left(\mathbf{j}^{*}-\mathbf{j}^{\prime *}\right)\right] \\
{\left[u_{\gamma^{\prime}}^{*}\left(\mathbf{j}^{\prime *}, t\right)-U_{0 \gamma^{\prime}} \delta(\mathbf{j})^{\prime *}\right]\left[u_{\gamma}^{*}\left(\mathbf{k}^{*}-\mathbf{j}^{*}, t\right)-U_{0 \gamma} \delta\left(\mathbf{k}^{*}-\mathbf{j}^{*}\right)\right]}
\end{gathered}
$$

Since $j^{*}$ is in the subgrid scale, while $j^{\prime *}$ and $k^{*}$ are in the supergrid, $\delta\left(\mathbf{k}^{*}-\mathbf{j}^{*}\right)$ and $\delta\left(\mathbf{j}^{*}-\mathbf{j}^{\prime *}\right)$ can never be simultaneously satisfied. As a result,

$$
\begin{gathered}
N S_{T}^{*}=2 M_{\alpha \beta \gamma}\left(k^{*}\right) \int d^{3} j^{*} d^{3} j^{\prime *}\left(\frac{j^{*}}{k_{c}}\right)^{4 / 3} \frac{M_{\alpha \beta \gamma}\left(j^{*}\right)}{\nu\left(k_{c}^{*}\right) j^{* 2}} u_{\beta^{\prime}}^{*}\left(\mathbf{j}^{*}-\mathbf{j}^{\prime *}, t\right) \\
{\left[u_{\gamma^{\prime}}^{*}\left(\mathbf{j}^{\prime *}, t\right)-U_{0 \gamma^{\prime}} \delta\left(\mathbf{j}^{\prime *}\right)\right] u_{\gamma}^{*}\left(\mathbf{k}^{*}-\mathbf{j}^{*}, t\right)}
\end{gathered}
$$


Now only one term in Eq. (34) could violate the Galilean invariance of the renormalized Navier-Stokes equation. However,

$$
\int d^{3} j^{\prime *} \delta\left(\mathbf{j}^{\prime *}\right) u_{\beta^{\prime}}^{*}\left(\mathbf{j}^{*}-\mathbf{j}^{\prime *}, t\right) \rightarrow u_{\beta^{\prime}}^{*}\left(\mathbf{j}^{*}, t\right)
$$

This is not permissible since $u_{\beta^{\prime}}^{*} \equiv u_{\beta^{\prime}}^{<*}$ and $j^{*}$ is restricted to the subgrid. Thus $N S_{T}=$ $N S_{T}^{*}$. Hence the triple term is Galilean invariant.

\subsection{Galilean invariance in the renormalized passive scalar equa- tion}

The renormalized passive scalar equation has two triple nonlinear terms. The proof of the Galilean invariance of the renormalized passive scalar equation proceeds in a similar manner to that for the renormalized Navier-Stokes equation.

For the first triple nonlinear term, labelled $\bar{P} S_{T 1}$, after a Galilean transformation, we found

$$
\begin{array}{r}
P S_{T 1}^{*} \sim \int d^{3} j^{*} d^{3} j^{\prime *}\left[u_{\alpha}^{<*}\left(\mathbf{k}^{*}-\mathbf{j}^{*}, t\right)+U_{0 \alpha} \delta\left(\mathbf{k}^{*}-\mathbf{j}^{*}\right)\right]\left[u_{\beta}^{<*}\left(\mathbf{j}^{*}-\mathbf{j}^{\prime *}, t\right)+U_{0 \beta} \delta\left(\mathbf{j}^{*}-\mathbf{j}^{\prime *}\right)\right] T^{<*}(\mathbf{j}, t) \\
=\int d^{3} j^{*} d^{3} u_{\alpha}^{<*}\left(\mathbf{k}^{*}-\mathbf{j}^{*}, t\right) u_{\beta}^{<*}\left(\mathbf{j}^{*}-\mathbf{j}^{\prime *}\right) T^{<*}(\mathbf{j}, t)(36)
\end{array}
$$

since $j^{*}$ is in the subgrid while $k^{*}, j^{\prime *}$ are in the resolvable scale. Thus the $\delta\left(\mathbf{k}^{*}-\mathbf{j}^{*}\right)$ and $\delta\left(\mathbf{j}^{*}-\mathbf{j}^{*}\right)$ can never be satisfied simultaneously.

The second triple nonlinear term has the following structure after the Galilean transformation

$$
\begin{array}{r}
P S_{T 2}^{*} \sim \int d^{3} j^{*} d^{3} j^{\prime *}\left[u_{\beta}^{<*}\left(\mathbf{j}^{\prime *}, t\right)+\right. \\
\left.U_{0 \beta} \delta\left(\mathbf{j}^{\prime *}\right)\right]\left[u_{\gamma}^{<*}\left(\mathbf{k}^{*}-\mathbf{j}^{*}-\mathbf{j}^{\prime *}, t\right)+U_{0 \gamma} \delta\left(\mathbf{k}^{*}-\mathbf{j}^{*}-\mathbf{j}^{\prime *}\right)\right] T^{<}(\mathbf{j}, t) \\
\rightarrow \int d^{3} j^{*} d^{3}\left[u_{\beta}^{<*}\left(\mathbf{j}^{* *}\right)+U_{0 \beta} \delta\left(\mathbf{j}^{\prime *}\right)\right]\left[u_{\gamma}^{<*}\left(\mathbf{k}^{*}-\mathbf{j}^{*}-\mathbf{j}^{\prime *}, t\right) T^{<}(\mathbf{j}, t)\right. \\
\rightarrow \int d^{3} j^{*} d^{3} u_{\beta}^{<*}\left(\mathbf{j}^{\prime *}, t\right) u_{\gamma}^{<*}\left(\mathbf{k}^{*}-\mathbf{j}^{*}-\mathbf{j}^{\prime *}, t\right) T^{<}(\mathbf{j}, t)(37)
\end{array}
$$

where the last two steps follow from the wavenumber constraints. $k, j, j^{\prime}$ are in the resolvable scales while $|\mathbf{k}-\mathbf{j}|$ is in the subgrid scale. Specifically, the first step follows since $\delta\left(\mathbf{k}^{*}-\mathbf{j}^{*}-\mathbf{j}^{\prime *}\right)$ can never be satisfied. The second step follows since $\delta\left(\mathbf{j}^{\prime *}\right)$ would force $u_{\gamma}^{<*}\left(\mathbf{k}^{*}-\mathbf{j}^{*}-\mathbf{j}^{\prime *}\right) \rightarrow u_{\gamma}^{<*}\left(\mathbf{k}^{*}-\mathbf{j}^{*}\right)$. This is not permissible since $|\mathbf{k}-\mathbf{j}|$ is in the subgrid while $u_{\gamma}^{*}$, by definition, is in the resolvable scale. Thus, the renormalized passive scalar equation is also Galilean invariant. 


\section{Differential equations for the renormalized eddy viscosity and diffusivity}

The differential limit, $f \rightarrow 1$, is singular and has been discussed recently ${ }^{7}$. In particular, it is related to the assumption of local versus non-local interactions in $k$. In this section we will calculate the eddy viscosity and diffusivity under the differential equation limit for recursive RNG.

For recursive RNG we will find that the differential equations hold throughout the resolvable wavenumber range $0<k \leq k_{c}$. This should be contrasted with $\epsilon$ - RNG eddy viscosity differential equation which is valid only in the $k \rightarrow 0 \mathrm{limit}^{8}$.

\subsection{The distance interaction approximation, $k \rightarrow 0$}

Consider the resolvable scale Navier-Stokes equation, Eq. (9),

$$
\begin{array}{r}
{\left[\frac{\partial}{\partial t}+\nu_{0} k^{2}\right] u_{\alpha}^{<}(\mathbf{k}, t)=M_{\alpha \beta \gamma}(k) \int d^{3} j\left[u_{\beta}^{<}(\mathbf{j}, t) u_{\gamma}^{<}(\mathbf{k}-\mathbf{j}, t)\right.} \\
\left.+2 u_{\beta}^{>}(\mathbf{j}, t) u_{\gamma}^{<}(\mathbf{k}-\mathbf{j}, t)+u_{\beta}^{>}(\mathbf{j}, t) u_{\gamma}^{>}(\mathbf{k}-\mathbf{j}, t)\right]
\end{array}
$$

The first and third terms on the RHS of (9) are symmetric in $j$ and $|\mathbf{k}-\mathbf{j}|$ in terms of their respective wavenumber constraints in wavenumbers. As a result, the distant interaction limit $k \rightarrow 0$ has no effect on the existence of these terms which will give rise to the standard quadratic nonlinearity and eddy viscosity, respectively. However, the second term on the RHS of (9) has the following constraint: $j$ is in the subgrid while $|\mathbf{k}-\mathbf{j}|$ is in the resolvable scales. Specifically, the consistency condition requires that, for small $k, j$ satisfies

$$
j>k_{c} \quad \text { and } \quad j<k_{c}+k z .
$$

Since $|z| \leq 1$, the range of integration must be $O(k)$.

Thus, the second term on the RHS of Eq. (9) can not contribute in the limit $k \rightarrow 0$ since the integrand is bounded. A similar conclusion can be drawn for the second and third terms in the renormalized passive scalar equation. Hence, the triple terms will not contribute to the renormalized momentum equations and recursion relation for the transport coefficients in the distant interaction limit, $k \rightarrow 0$. However, they will contribute to the renormalized Navier-Stokes and passive scalar equations for $0<k \leq k_{c}$. 


\subsection{Test of the conclusion in $\S 4.1$ from numerical simulation databases}

The conclusions of the last subsection can be tested directly using numerical simulation databases. Indeed, energy transfer and eddy viscosity can be analysed using results from numerical simulations by introducing an artificial cut at a wavenumber $k_{c}$ that is smaller than the maximum resolved wavenumber $k_{m}$ of the simulation. With this fictitious separation between the subgrid and resolvable scales, it is possible to evaluate the effect of the subgrid $k_{c}<k<k_{m}$ on the resolvable scales $k_{c}$. To facilitate comparison with the recursive $\mathrm{RNG}$ analysis, we consider separately the contribution to the energy transfer and eddy viscosity from the second and third term on the RHS of Eq. 9. We form an energy equation from the momentum equation and introduce the following notation: $T^{<<(k)}$ is the spectrum of energy transfer to mode $\mathrm{k}$ resulting from interactions between modes with wavenumbers less than $k_{c} ; T^{><}(k)$ and $T^{>>}(k)$ represent similar contributions from interactions with one or both modes above the cutoff $k_{c}$, respectively. The equivalent contributions to eddy viscosity in the energy equations are $\nu^{><}(k)=-T^{><}(k) / 2 k^{2} E(k)$, and $\nu^{>>}(k)=-T^{>>}(k) / 2 k^{2} E(k)$.

To determine the behavior of the energy transfer and eddy viscosity $\nu^{><(k) \text { and } \nu>>(k)}$ we measured them in flow fields obtained from numerical simulations on $128^{3}$ meshes of forced turbulence. The forced flow dataset was generated by Chasnov ${ }^{15}$ in a large-eddy simulation (LES) of the Kolmogorov inertial range, using a subgrid model derived from the stochastic equation that is consistent with Eddy-damped-quasinormal Markovian (EDQNM) approximation.

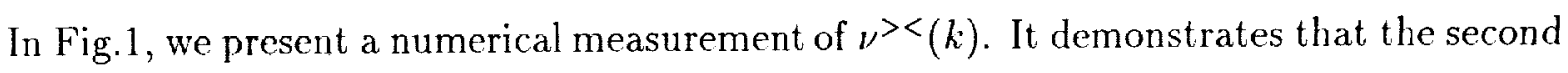
term on the RISS of Eq. (9) does not contribute to the energy transfer process as $k \rightarrow 0$, consistent with our analysis.

\subsection{The differential equation limit, $f \rightarrow 1$}

We now derive the differential equation for the transport coefficients for finite $k, 0<k \leq 1$, where the wavenumbers are normalized with respect to the cutoff wavenumber $k_{c}=1$. The o.d.e. in the distant interaction limit $(k=0)$ will be derived in the next subsection. After the rescaling, we rewrite the recursion relation in the form

$$
\nu_{n+1}(k)-f^{(y+1) / 3} \nu_{n}(f k)=f^{(y+1) / 3} \delta \nu_{n}(f k)
$$

For $f \rightarrow 1$, the number of iterations $n \rightarrow \infty$. Similarity considerations lead to

$$
\nu_{n+1}(k) \rightarrow \nu(k), \quad n \rightarrow \infty
$$


Let $\Delta=1-f$. the LHS of Eq. (39) becomes

$$
\nu(k)-[1-\Delta]^{(y+1) / 3} \nu[k(1-\Delta)] \rightarrow \Delta\left[\frac{d \nu(k)}{d k}+\frac{y+1}{2} \nu(k)+O(\Delta)\right]
$$

As noted earlier ${ }^{7}$, the partial average of $\operatorname{Rose}^{6}$ must be employed in order to insure the existence of the differential limit. The partial average is introduced since the distinction between the resolvable and subgrid scales become fuzzy in the limit of a differential subgrid partitioning, $f \rightarrow 1$.

Following Rose ${ }^{6}$, we first change the variable from $j, z$ to $j, q=|\mathbf{k}-\mathbf{j}|$, with $d j d z=$ $(q / k j) d j d q$, so that the RHS of Eq. (39) becomes

$$
\begin{array}{r}
\delta \nu(k)=\int d j d q\left(\frac{q}{k j}\right) \frac{L(k, j, q)}{\nu(j) k^{2} \nu(|\mathbf{k}-\mathbf{j}|)|\mathbf{k}-\mathbf{j}|^{2}|\mathbf{k}-\mathbf{j}|^{y}} \\
+\int d j d q\left(\frac{q}{k j}\right) \frac{L(k, j, q)}{\nu(j) k^{2} \nu(|\mathbf{k}-\mathbf{j}|)|\mathbf{k}-\mathbf{j}|^{2}|\mathbf{k}-\mathbf{j}|^{y}}\left(\frac{j}{k_{c}}\right)^{(y+1) / 3} \\
\rightarrow \Delta \int_{1<q<1+k} d q \frac{L(k, 1, q)}{k^{3} \nu^{2}(1) q^{y-1}}+\Delta \int_{1<j<1+k} d j \frac{L(k, j, 1)}{k^{3} \nu^{2}(1)} j^{(y-2) / 3}
\end{array}
$$

where

$$
L(k, 1, q)=k\left(1-z^{2}\right)\left(k-z q^{2}\right) / q^{2}
$$

and

$$
L(k, j, 1)=k j\left(1-z^{2}\right)(k j-z) .
$$

Here one has set the coefficient $D_{0}^{\prime}=2 \pi D_{0}=1$ (Zhou et al. ${ }^{11}$ ).

As a result, the fixed point renormalized eddy viscosity $\nu(k)$ is determined from the o.d.e. at $O(\Delta)$

$$
k \frac{d \nu(k)}{d k}+\frac{y+1}{3} \nu(k)=\frac{1}{\nu^{2}(1)}\left[A_{\nu}(k)+B_{\nu}(k)\right]
$$

where

$$
\begin{gathered}
A_{\nu}(k)=\frac{1}{k^{3}} \int_{1}^{1+k} d q \frac{L(k, 1, q)}{q^{y-1}} \\
B_{\nu}(k)=\frac{1}{k^{3}} \int_{1}^{1+k} d j L(k, j, 1) j^{(y-2) / 3}
\end{gathered}
$$

Here, $z$ is evaluated at $j=1$ and $q=1$, respectively in the $L(k, j, q)$ expression.

The fixed point o.d.e. for the eddy diffusivity is that given by 


$$
k \frac{d \mu(k)}{d k}+\frac{y+1}{3} \mu(k)=\frac{1}{\mu(1)}\left[A_{\mu}(k)+B_{\mu}(k)\right]
$$

where

$$
\begin{gathered}
A_{\mu}(k)=\frac{1}{2 k} \int_{1 \leq q \leq 1+k} d q \frac{\sin ^{2}(k, q, 1)}{q^{m+1}} \\
B_{\mu}(k)=\frac{1}{2 k} \int_{1 \leq j \leq 1+k} d j \sin ^{2}(k, 1, j) j^{(m-1) / 2}
\end{gathered}
$$

and $\sin ^{2}(k, j, q)$ is the square of the sine of the angle defined by the $k$ and $q$ legs of the $\mathbf{k}, \mathbf{j}, \mathbf{q}$ wave-vector triangle. Note that Eqs. (48)-(50) are identical with the o.d.e. of eddy diffusivity which Rose $^{6}$ derived for a prescribed frozen velocity field.

\subsection{Differential equations in the $k \rightarrow 0$ limit}

In the $k \rightarrow 0$, we have seen that the triple term does not contribute to the eddy viscosity. As a result, the recursion relation will now contain only the usual quadratic contribution. We further simply the analysis by taking the standard subgrid linear propagator ${ }^{11} G_{h}^{-1}(|\mathbf{k}-\mathbf{j}|)=$ $\left[\frac{\partial}{\partial t}+\nu_{h}(|\mathbf{k}-\mathbf{j}|)\right] \sim G_{h}^{-1}(|\mathbf{j}|)$ as $k \rightarrow 0$.

The limits of the integration are given by

$$
1<j-k z<1 / f, \quad 1+k z<j<1 / f+k z
$$

Thus, the RHS of Eq. (39) becomes

$$
\delta \nu_{n}(k)=M-A-B
$$

where the integral limits for these terms are

$$
\begin{array}{lll}
\int_{1}^{1 / f} d j \int_{-1}^{1} d z & \text { for } & M \\
\int_{0}^{1} d z \int_{1}^{1+k z} d j & \text { for } & A \\
\int_{-1}^{0} d z \int_{1 / f+k z}^{1 / f} d j & \text { for } & B .
\end{array}
$$

Terms $A$ and $B$ are the corrections to the symmetric term $M$. They are important for a finite bandwidth $f$. However, it is easy to show that $A+B=0$ for $f \rightarrow 1$ in the $k \rightarrow 0$ limit. Hence

$$
\delta \nu_{n}^{D}(k) \rightarrow-\Delta \frac{D_{0}^{\prime}}{k \nu^{2}(1)}\left[\frac{1}{j^{y+1}}\right]_{j=1} \int_{-1}^{1} d z\left[1-z^{2}\right]\left[z+\frac{k}{j}\left(y z^{2}-1\right)\right]=\Delta \frac{8}{15} \frac{D_{0}^{\prime}}{\nu^{2}(1)}
$$


while the LHS of Eq. (39) yields

$$
k \frac{d \nu(k)}{d k}+\frac{y+1}{3} \nu(k) \rightarrow \frac{y+1}{3} \nu(k), \quad \text { as } \quad k \rightarrow 0
$$

since $\frac{d \nu(k)}{d k}$ is bounded as $k \rightarrow 0$.

Thus, as $k \rightarrow 0$,

$$
\nu(k \rightarrow 0)=\frac{3}{y+1} \frac{8}{15} \frac{D_{0}^{\prime}}{\nu^{2}(1)} .
$$

Again, one sets the coefficient $D_{0}^{\prime}=2 \pi D_{0}=1$.

A similar anlysis can be performed on the fixed point o.d.e for the eddy diffusivity. This was not performed by Rose ${ }^{6}$ who did not consider the $k \rightarrow 0$ limit carefully. Again, as $k \rightarrow 0$, the triple term will not contribute and we find that the corresponding $\delta \mu^{D}(k)$ term has the limiting form

$$
\begin{aligned}
\delta \mu^{D}(k) \rightarrow \Delta \frac{1}{2 \mu(1)}\left[\frac{1}{j^{11 / 3}}\right] \int d z\left[1+\left(\frac{17}{3}\right) \frac{k z}{j}\right]\left(1-z^{2}\right)_{p=1} & =\Delta \frac{1}{2 \mu(1)} \int_{-1}^{1} d z\left(1-z^{2}\right) \\
& =\Delta \frac{2}{3 \mu(1)}
\end{aligned}
$$

as $k \rightarrow 0$. Hence

$$
\mu(k \rightarrow 0)=\frac{2}{y+1} \frac{1}{\mu(1)} .
$$

\subsection{The momentum equation eddy viscosity and diffusivity}

The o.d.e's, Eqs. (45) and (48), for the momentum equation eddy viscosity and diffusivity are readily solved and shown in Fig. 2. We observe that both the eddy viscosity and diffusivity have a similar plateau structure as $k \rightarrow 0$. Notice that the eddy diffusivity platcau is not obtained in the original numerical calculation of Rose $\mathrm{R}^{6}$. As $k \rightarrow k_{c}$, eddy viscosity displays a weak cusp like behavior while the eddy diffusivity decreases monotonically as $k \rightarrow k_{c}$. These curves, as $k \rightarrow k_{c}$, are similar to that of Zhou et al. ${ }^{11}$ and Rose ${ }^{6}$.

\section{The turbulent transport coefficient in the second moments}

The concept of the spectral eddy viscosity and diffusivity are introduced in the second moments ${ }^{3,4}$. Thus, the momentum equation spectral eddy viscosity and diffusivity are only 
a partial contribution to the total transport coefficients. Indeed from our numerical measurement in Fig. 1, we expect that the triple nonlinear terms will contribute to the cnergy transfer when $k$ is near $k_{c}$.

We consider the contribution of the triple nonlinear term in the renormalized eddy viscosity to the eddy viscosity first (Zhou and Vahala ${ }^{16}$ ). The second moment for the velocity field is defined as

$$
U_{\alpha \beta}(\mathbf{k}, t) \equiv<u_{\alpha}(\mathbf{k}, t) u_{\beta}(-\mathbf{k}, t)>
$$

The time evolution of $U_{\alpha \beta}(\mathbf{k}, t)$ is

$$
\frac{\partial U_{\alpha \beta}(\mathbf{k}, t)}{\partial t}=-2 \nu(k) k^{2} U_{\alpha \beta}(\mathbf{k}, t)+2<f_{\alpha}(\mathbf{k}, t) u_{\beta}(-\mathbf{k}, t)>+T_{\alpha \beta}^{D}(\mathbf{k}, t)+T_{\alpha \beta}^{T}(\mathbf{k}, t)
$$

In this equation, $T_{\alpha \beta}^{D}(\mathrm{k}, t)$ is the standard energy transfer from the quadratic nonlinearity. In contrast, $T_{\alpha \beta}^{T}(\mathbf{k}, t)=-2 \nu_{T}(k) k^{2} E(k)$ is the energy transfer arising from the RNG induced triple nonlinearity. It is readily shown that ${ }^{16}$

$$
\nu_{T}(k)=\frac{1}{2 \nu\left(k_{c}\right) k_{c}^{(m+1 / 2)}} \frac{1}{k^{2}} \int_{k_{c}}^{k+k_{c}} d j d z \frac{L(k, j, q)|\mathbf{k}-\mathbf{j}|^{-y-2} j^{y+1 / 3}}{\nu(\mathbf{k}-\mathbf{j})} .
$$

In Fig. 3, we see that $\nu_{T}(k)$ is the major contributor to the cusp-like behavior of the spectral eddy viscosity as $k \rightarrow 0$. For $k \ll 1$, it has a backscatter of energy from the subgrid and resolvable scales.

We now define $\Theta(k)$ as the scalar variance $\Theta(k) \equiv<T(-k, t) T(k, t)>$. The dynamic equation for $\Theta(k)$ can be constructed by multiplying Eq. (25) with $T(-k, t)$, followed by an averaging operation. Again, a quasi-normal approximation is applied to reduce the fourth moment to the product of the second moments. Notice that the last term on the RHS of Eq. (25) will not contribute to the spectral equation since $<u_{\beta}\left(\mathbf{j}^{\prime}, t\right) u_{\gamma}\left(\mathbf{k}-\mathbf{j}-\mathbf{j}^{\prime}, t\right)>\sim \delta(\mathbf{k}-\mathbf{j})$, a condition that can not be satisfied since $k-j$ is in the subgrid scale.

The dynamical equation for the scalar variance is

$$
\left[\frac{\partial}{\partial t}+\mu(k) k^{2}\right] \Theta(k, t)=\Sigma^{D}+\Sigma^{T}
$$

where $\Sigma^{D}$ is the usual transfer function for the passive scalar. $\Sigma^{T}(k)$ is the additional contribution from the triple nonlinear term induced by the recursive RNG procedure

$$
\begin{aligned}
\Sigma^{T}(k)=-\frac{2 k_{\alpha}}{\mu\left(k_{c}\right) k_{c}^{m+1 / 2}} \int d^{3} j d^{3} j^{\prime} \frac{j_{\beta}^{\prime}}{j^{3-m / 2}}<u_{\alpha}(\mathbf{k}-\mathbf{j}) u_{\beta}\left(\mathbf{j}-\mathbf{j}^{\prime}\right) & ><T\left(\mathbf{j}^{\prime}\right) T(-\mathbf{k})> \\
& =-2 \mu_{T}(k) k^{2} \Theta(k, t)
\end{aligned}
$$


where

$$
\mu_{T}(k)=\frac{1}{\mu\left(k_{c}\right) k_{c}^{m+1 / 2}} \frac{k_{\alpha} k_{\beta}}{k^{2}} \int d^{3} j \frac{D_{\alpha \beta}(k-j) Q(|\mathbf{k}-\mathbf{j}|)}{j^{3-m / 2}}
$$

and the incompressible condition has been used. It is seen in Fig. 4 that $\mu_{T}(k)$ is small when $k$ is small. However, as $k \rightarrow k_{c}, \mu_{T}(k)$ increases rapidly.

The solution of $\nu_{T}(k)$ is very similar to that of $\mu_{T}(k)$ as $k \rightarrow k_{c}$. They are the major contribution to the strong cusp in the eddy viscosity found from the Test Field model ${ }^{3}$ and EDQNM ${ }^{4}$. Furthermore, $\nu_{T}(k)$ also contains the backscatter of the energy from the subgrid to the resolvable scale. This is a major difference between $\mu_{T}(k)$ and $\nu_{T}(k)$.

Rose $^{6}$ discussed the role of the triple nonlinear terms in physical space. He pointed out that it represents the possibility of an exchange of scalar eddies between the resolvable and subgrid scales. This effect is an inherent property of measurements made on the passive scalar system with instruments which have a spatial resolution limited to an eddy width size greater than $1 / k_{c}$.

\section{Spectral eddy viscosity, diffusivity and Prandtl num- ber}

The spectral eddy viscosity is simply the sum of the contributions from the momentum equation and the triple nonlinear term. The result is presented in Fig. 5. It appears that our calulation is in qualitative agreement with the closure theory ${ }^{3,4}$ and direct numerical measurements ${ }^{14-18}$. In particular, it predicts the correct asymptotic behaviors of the eddy viscosity as $k \rightarrow 0$ and $k \rightarrow k_{c}$ (Kraichnan ${ }^{3}$ ).

Our spectral eddy diffusivity shows a plateau at $k \rightarrow 0$, in good agreement with the EDQNM calculation of Chollet ${ }^{4}$. However, the EDQNM calculation is not unique and depends on the choice of the parameters. Our diffusivity is in good agreement with EDQNM when parameters are chosen according to the direct interaction approximation (DIA ${ }^{4}$.

The spectral Prandtl number can be easily determined from our calculated eddy viscosity and diffusivity (Fig. 5). It is a function of $k$ and has values ranging from $0.72 \sim 0.92$. Note that our turbulent Prandtl number at $k \rightarrow 0$ limit is very close to that reported by Yakhot and Orszag $^{8}$ (0.7179). The values of turbulent Prandtl numbers found experimentally 19 in the boundary layer are in the range of $0.6 \sim 0.8$. Hinze ${ }^{20}$ and Tennekes and Lumley ${ }^{21}$ pointed out that the transfer of passive scalar and velocity fields may be equally effective. Thus the turbulent Prandtl number is about 1 . However, Lesieur and Rogallo ${ }^{18}$ found that their spectral Prandtl number only rose from 0.2 at small $k$ to 0.8 near the cutoff. Lesieur ${ }^{14}$ recently found that the turbulent Prandtl number may be much closer to 1 than that of 
Lesieur and Rogallo ${ }^{18}$. The EDQNM spectral Prandtl number depends on the choice of two EDQNM closure scalar adjustable constants ${ }^{4,14}$. In the first case, it remains approximately cqual to 0.6 , even in the vicinity of $k_{c}$. In the second case, it has a plateau value of 0.33 , and a cusp close to $k_{c}$, where it rose to 0.6 .

\section{Discussion and conclusions}

In this paper we have applied recursive RNG to the problem of the advection of a passive scalar by incompressible turbulence. We have clarified the role of the higher-order RNG induced nonlinearities and shown that: (a) The renormalized evolution equations are still Galilean invariant (i.e., these higher-order nonlinearities do not destroy the Galilean invariance of the original equations). This is an important property that needs to be preserved in subgrid modeling, especially as one proceeds to more complicated flows and boundaries. (b) These higher-order nonlinearities do not contribute to the transport coefficients as $k \rightarrow 0$.

Now the typical byproduct of the recursive RNG methods is a complicated integrodifference recursion relation to be solved for the eddy transport coefficients $\mathrm{s}^{6,10-11}$. This recursion relation is a function of the subgrid shell thickness parameter $f$. Here, we have shown how to pass to the differential subgrid shell thickness limit $f \rightarrow 1$. In this limit, we recover an ordinary differential equation for the eddy coefficients - an equation that is very easily solved.

The o.d.e that is derived in recursive-RNG is fundamentally different from that derived by $t-R N G$ techniques. For convenience, we have summarized the Yahkot-Orszag derivation of the $\epsilon$-RNG o.d.e in the Appendix. In $\epsilon$-RNG, one is forced into taking the $k \rightarrow 0$ limit $^{8-9}$, and the independent variable of the resulting o.d.e is actually the cut-off wavenumber $k_{c}$. In recursive $\mathrm{RNG}$, the independent variable is the resolvable scale wavenumber $k, 0<k \leq k_{c}$, with a renormalization transformation that permits $k_{c}$ to be fixed. There is no renormalization transformation made in the Yakhot-Orszag $\epsilon$-RNG formulation. In the limit $k \rightarrow 0$, the eddy transport coefficients from both theories are in very close agreement. This is to be expected since the higher-order recursive RNG-induced nonlinearities $\rightarrow 0$ as $k \rightarrow 0$. The slight difference in the eddy coefficients (in the $k \rightarrow 0$ ) between the two theories can be attributed to the treatment of $k_{c}$ : i.e., whether one performs $\mathrm{RNG}$ rescaling transformations (recursive RNG) or not. The important effect of the triple nonlinearities introduced by the recursive RNG procedure are clearly seen in the second moment equations - especially for a resolvable wavenumber $k \rightarrow k_{c}$.

The spectral eddy viscosity, diffusivity and Prandtl number are determined and we find good agreement with both closure theory $y^{3-4}$ and direct numerical simulations ${ }^{14-16}$. 


\section{Appendix: Brief summary of the Yakhot-Orszag's derivation of o.d.e. for the eddy viscosity}

In the $\epsilon$ - RNG, a small parameter $\epsilon$ is introduced into the exponent of the forcing correlation function, Eq. (4),

$$
\epsilon=4+y-d=1+y
$$

where $d=3$ is the dimensionality ${ }^{8}$.

The distance interaction ${ }^{9}$ is introduced at the outset ${ }^{8}, k \rightarrow 0$. Thus, the triple nonlinearities do not contribute to the recursion relation, Eq. (14). Furthermore, the eddy viscosity is a function of the cutoff wavenumber $k_{c}$ and is obviously independent of the resolvable scale wavenumber $0<k \leq k_{c}$. To emphasize this, we shall follow Smith and Reynolds ${ }^{22}$ and denote their eddy viscosity by $\nu\left(k \rightarrow 0, k_{c}\right)$.

By variation of the cutoff wavenumber $k_{c}$, Yakhot and Orszag $^{8}$ derived an o.d.e for $\nu\left(k \rightarrow 0, k_{c}\right)$

$$
\frac{d \nu\left(k \rightarrow 0, k_{c}\right)}{d k_{c}}=A_{3} \nu\left(k \rightarrow 0, k_{c}\right) \bar{\lambda}^{2}\left(k \rightarrow 0, k_{c}\right)
$$

where

$$
A_{3}=\frac{\tilde{A}_{3} S_{3}}{(2 \pi)^{3}}, \quad \tilde{A}_{3}=\frac{6-\epsilon}{30} \quad \bar{\lambda}\left(k \rightarrow 0, k_{c}\right)=\frac{D_{0}}{\left[\nu\left(k \rightarrow 0, k_{c}\right)\right]^{3 / 2} k_{c}^{c / 2}}
$$

where $S_{3}=4 \pi^{2}$ is the area of a unit sphere in three-dimensions. These equation should be compared with that Eqs. (43)-(45) where the resolvable scale wavenumber $k$ is the variable in the recursion relation derived from the recursive RNG, and not the cutoff $k_{c}$.

For a given boundary condition $\nu\left(k \rightarrow 0, k_{0}\right)$, an analytical solution can be obtained from Eqs. (A1-A3).

$$
\nu\left(k \rightarrow 0, k_{c}\right)^{3}-\nu\left(k \rightarrow 0, k_{0}\right)^{3}=\frac{3 A_{3} D_{0} S_{3}}{(2 \pi)^{3}} \frac{k_{c}^{-c}-k_{0}^{-\epsilon}}{\epsilon}
$$

where $k_{0}$ is typically in the order of Kolmogorov dissipation wavenumber. Eq. (A4) reduces to the familiar inertial range form by taking $\nu\left(k \rightarrow 0, k_{0}\right) \rightarrow \nu_{0}$ for $k_{c} \rightarrow k_{d}$. In that limit, Eq. (A4) takes the form

$$
\nu\left(k \rightarrow 0, k_{c}\right) \sim\left[\frac{2 S_{3} D_{0}}{(2 \pi)^{3}}\right]^{1 / 3}\left[\frac{3 \tilde{A}_{3}}{2 \epsilon}\right]^{1 / 3}
$$

where $\nu\left(k \rightarrow 0, k_{0}\right)$ is neglected since $\nu\left(k \rightarrow 0, k_{c}\right)>>\nu\left(k \rightarrow 0, k_{0}\right)$ in the fully-developed turbulence. 
In order to compare with the inertial range theory of Kraichnan ${ }^{23}$, Yakhot and Orszag set

$$
k_{\varepsilon}=k
$$

for any $k \in\left[0, k_{c}\right]$. From the closure theory analysis ${ }^{3,4}$ and direct numerical simulation measurements ${ }^{14-18}$, eddy viscosity has rather distinct characteristics in the limits $k \rightarrow 0$ and $k \rightarrow k_{c}$. As a result, Eq. (A6) is a rather oversimplified approximation ${ }^{22}$.

\section{Acknowledgments}

This research was supported by the National Aeronautics and Space Administration while the authors were in residence at the Institute for Computer Applications in Science and Engineering, NASA Langley Research Center. Authors wish to thank Dr. M.Y. Hussaini for his encouragement, and Dr. C. Speziale for stimulating discussions on the subject related to the Galilean invariance. One of us (Y.Z.) would like to thank Dr. F. Wallefe and Dr. L. Smith for communicating their unpublished report. 


\section{References}

1. G.T. Csanady, Turbulent diffusion in the Environment, (D. Reidel, Boston, 1973).

2. L. Landau and E.M. Lifshitz, Fluid Mechanics (Pergamon, NY, 1982).

3. R.H. Kraichnan, Eddy viscosity in two and three dimensions, J. Atmos. Sci., 33, 1521 (1976).

4. J.P. Chollet, Two-point closures as a subgrid scale modeling for large-eddy simulations, in Turbulent shear flows $I V$, edited by F. Durst and B. Launder, Lecture Notes in Physics, (Springer, 1984).

5. D. Forster, D. Nelson, and M. Stephen, Large-distance and long-time properties of a randomly stirred fluid, Phys. Rev., A 16, 732 (1977).

6. H.A. Rose, Eddy diffusivity, eddy noise, and subgrid-scale modelling, J. Fluid Mech., $\mathbf{8 1}, 719(1977)$

7. Y. Zhou and G. Vahala, Local interaction in renormalization methods for Navier-Stokes turbulence, Phys. Rev., A 46, 1136 (1992).

8. V. Yakhot and S. Orszag, Renormalization group analysis of turbulence I. theory, $J$. Sci.Compt., 1, 3 (1986).

9. R.H. Kraichnan, An interpretation of the Yakhot-Orszag turbulence theory, Phys. Flu$i d s, 30,2400$ (1987).

10. Y. Zhou, G. Vahala, and M. Hossain, Renormalization group theory for eddy-viscosity in subgrid modeling, Phys. Rev., A 37, 2590 (1988)

11. Y. Zhou, G. Vahala, and M. Hossain, Renormalized eddy viscosity and Kolmogorov's constant in forced Navier-Stokes turbulence, Phys. Rev., A 40, 5865 (1989).

12. C.G. Speziale, Galilean invariance of subgrid-scale stress models in the large-eddy simulation of turbulence, J. Fluid Mech., 156, 55 (1985)

13. M. Hossain, Turbulent transport of a passive scalar field by using a renormalization group method, Phys. Rev. A, (Dec. 1992).

14. M. Lesieur, Turbulence in Fluids, (Kluwer, Dordrecht, 1990) 
15. J. R. Chasnov, Simulation of the Kolmogorov inertial range using an improved subgrid model, Phys. Fluids, A 3, 188, 1991.

16. Y. Zhou and G. Vahala, Reformulation of recursive renormalization group based subgrid modeling of turbulence, Phys. Rev. A (in press, 1993)

17. J.A. Domaradski, R.W. Metcalfe, R.S. Rogallo, and J.J. Riley, Analysis of subgridscale eddy viscosity with use of results from direct numerical simulations, Phys. Rev. Lett., 58, 547 (1987)

18. M. Lesieur and R.S. Rogallo, Large-eddy simulation of passive scalar diffusion in isotropic turbulence, Phys. Fluids, A 1, 718 (1989).

19. L. Fulachier and R. Dumas, Spectral analogy between temperature and velocity fluctuations in a turbulent boundary layer, J. Fluid Mech., 77, 257 (1976)

20. J.O. Hinze, Turbulence, McGraw Hill (1957)

21. H. Tennekees, and J.L. Lumley, A First Course in Turbulence, MIT press, 1972.

22. L.M. Smith and W.C. Reynolds, On the Yakhot-Orszag renormalization group method for deriving turbulence statistics and models, Phys. Fluids, A 4, 364 (1992)

23. R.H. Kraichnan, Inertial range transfer in two and three-dimensional turbulence, $J$. Fluid Mech, , 47, 525 (1971) 


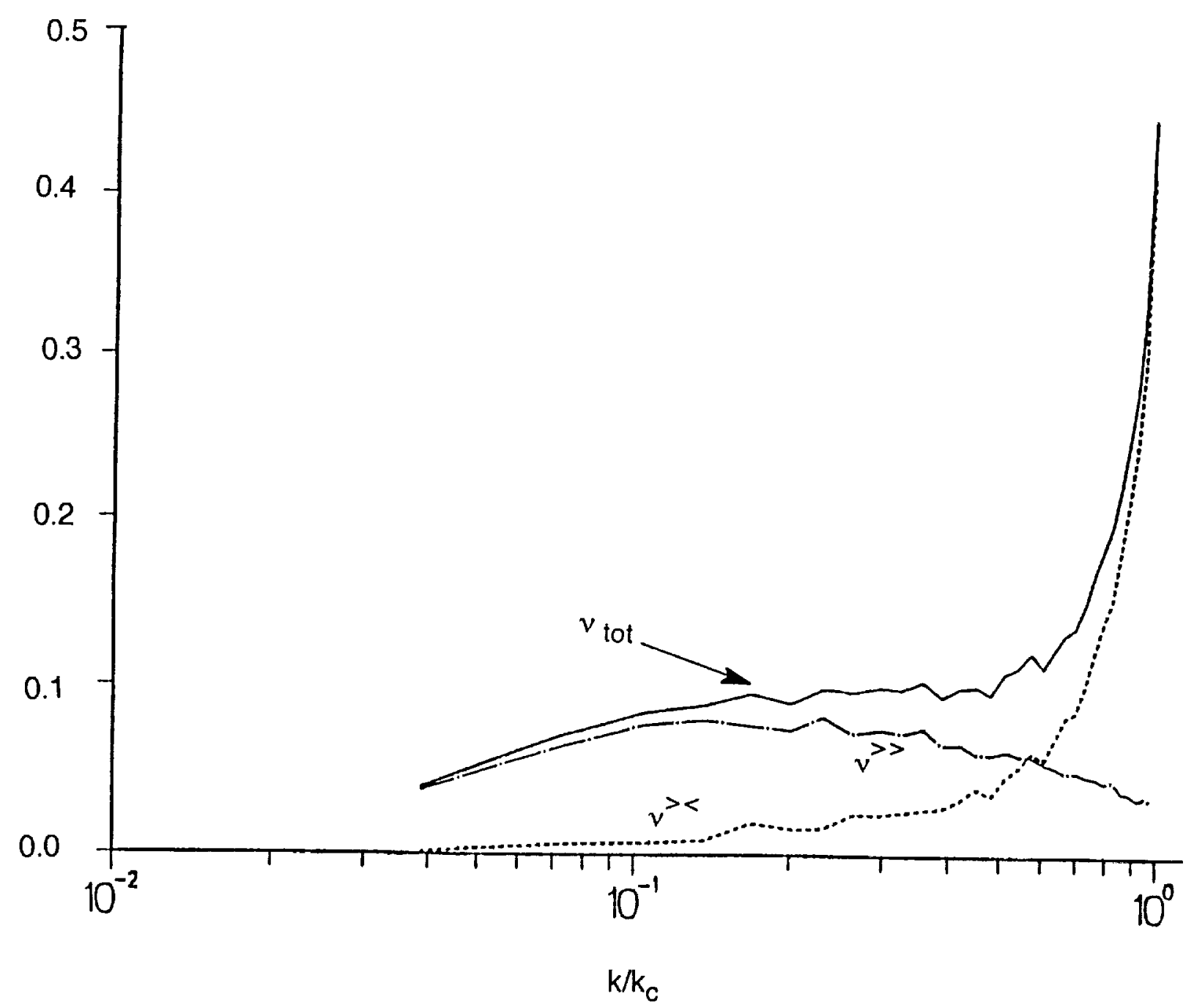

Fig. 1 Forced eddy viscosity profiles determined from LES databases for the fluid velocity at one time instant. $\nu_{\text {tot }}(k)=\nu^{>>}(k)+\nu^{><}(k)$, where $\nu^{>>}(k)$ arises from measured LES nonlocal subgrid energy transfer, and $\nu^{><}(k)$ arises from measured LES local subgrid energy transfer. It is important to note that $\nu^{>}<(k) \rightarrow 0$ as $k \rightarrow 0$ and that $\nu^{><}(k)$ arises from the $u^{<-u>}$ interaction. Note also the cusp bahvior in $\nu^{><}(k)$ as $k \rightarrow k_{c}$. 


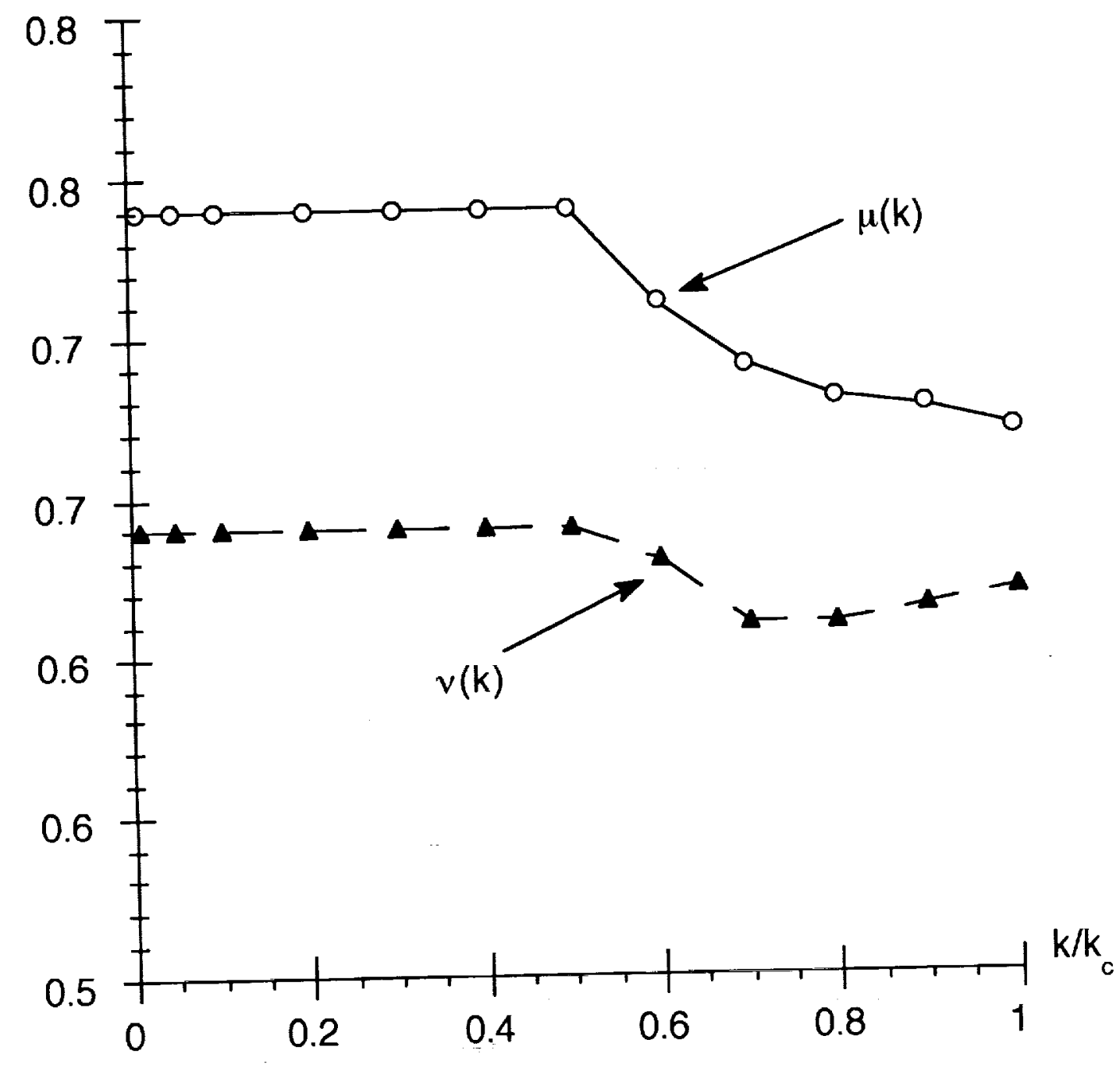

Fig. 2 A plot of the momentum eddy viscosity, $\nu(k)$, and diffusivity, $\mu(k)$, as a function of the resolvable scales, $0<k / k_{c}<1$. These profiles are determined from the o.d.e's for recursive RNG in the limit of differential subgrid shell thickness, $f \rightarrow 1$. 


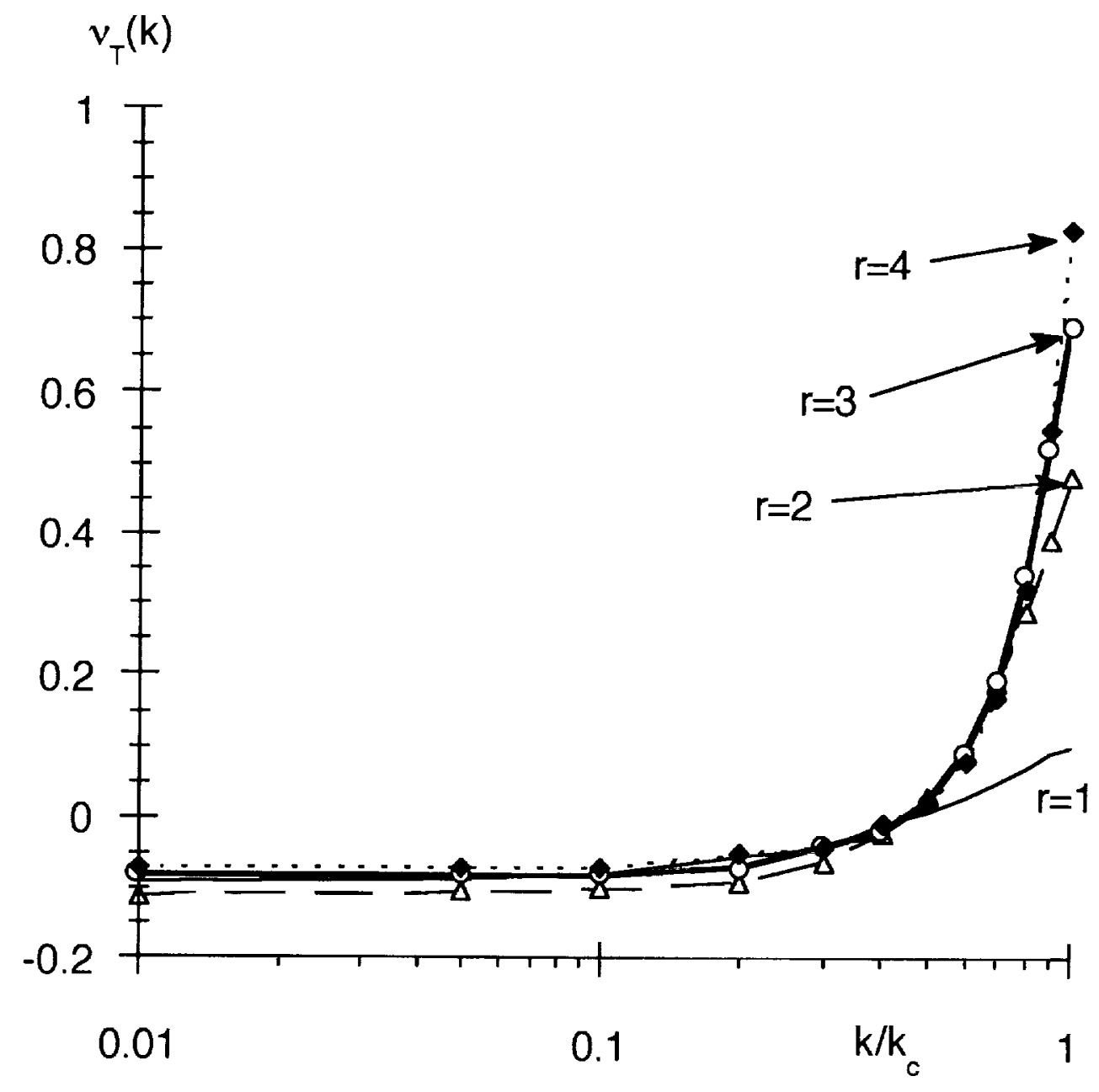

Fig. 3 The drain eddy viscosity $\nu_{T}(k)$ arising from the triple nonlinearities in the differential subgrid shell limit in recursive RNG. $r=k_{c} / K_{p}$ is a parameter in the productiontype energy spectrum, so that $E(k) \rightarrow k^{4}$ as $k \rightarrow 0 . K_{p}$ is a parameter that controls the location of the peak in $E(k)$. As $r$ increases, this peak in $E(k)$ moves to smaller $k$. Backscatter of energy from the subgrid scales to the large spatial scales is seen for $k / k_{c}<0.4$, the region in which $\nu_{T}(k)<0$. For $r>1$, there is a sharp cusp as $k \rightarrow k_{c}$. 


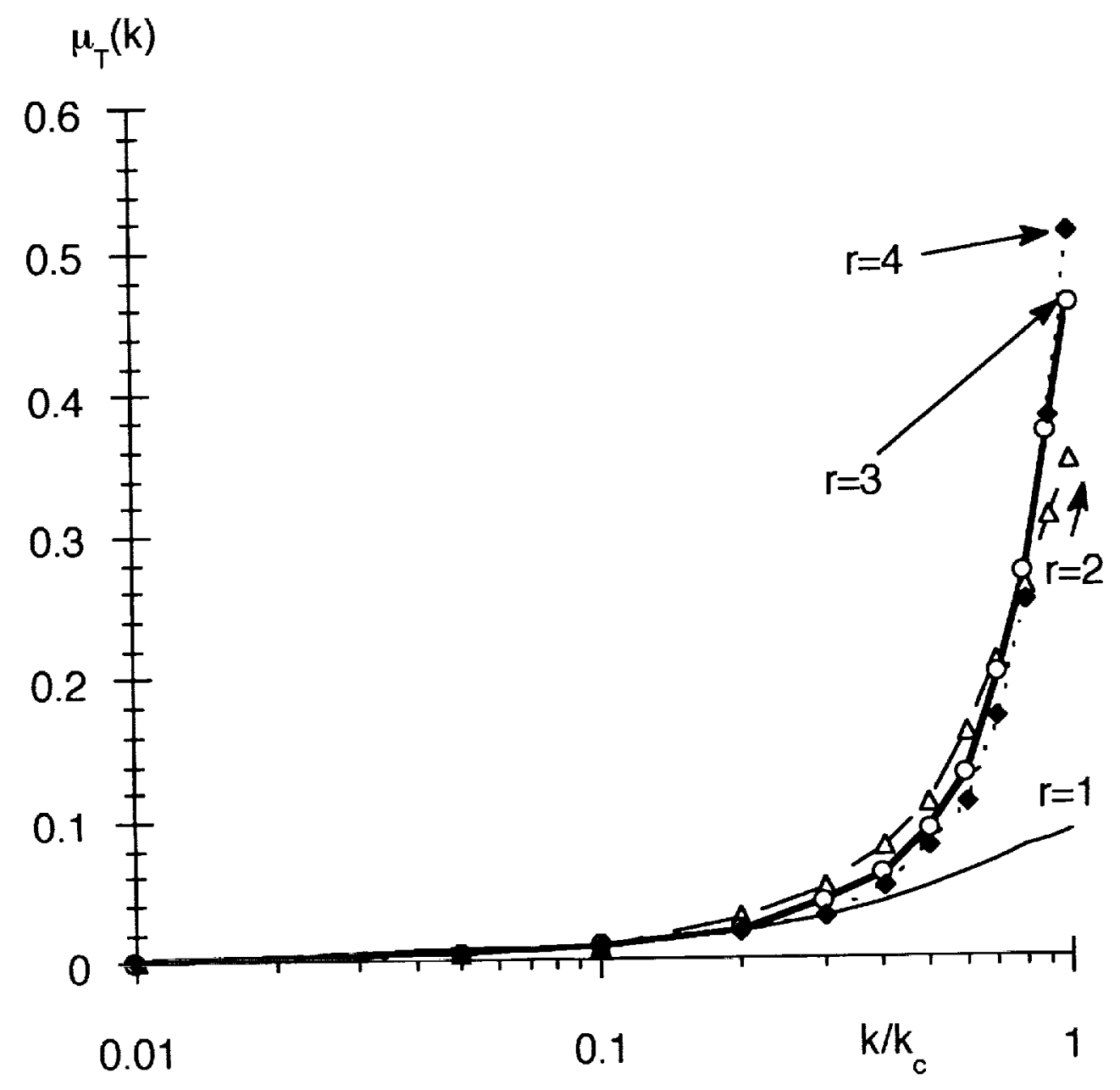

Fig. 4 The drain eddy diffusivity $\mu_{T}(k)$ arising from the triple nonlinearities in the differential subgrid shell limit of the scalar variance RNG evolution equation. The parameter $r$ is as in Fig. 3. Notice that there is now no backscatter of scalar variance, since $\mu_{T}(k)$ is non-negative for all $k$. There is a strong cusp as $k \rightarrow k_{c}$. 


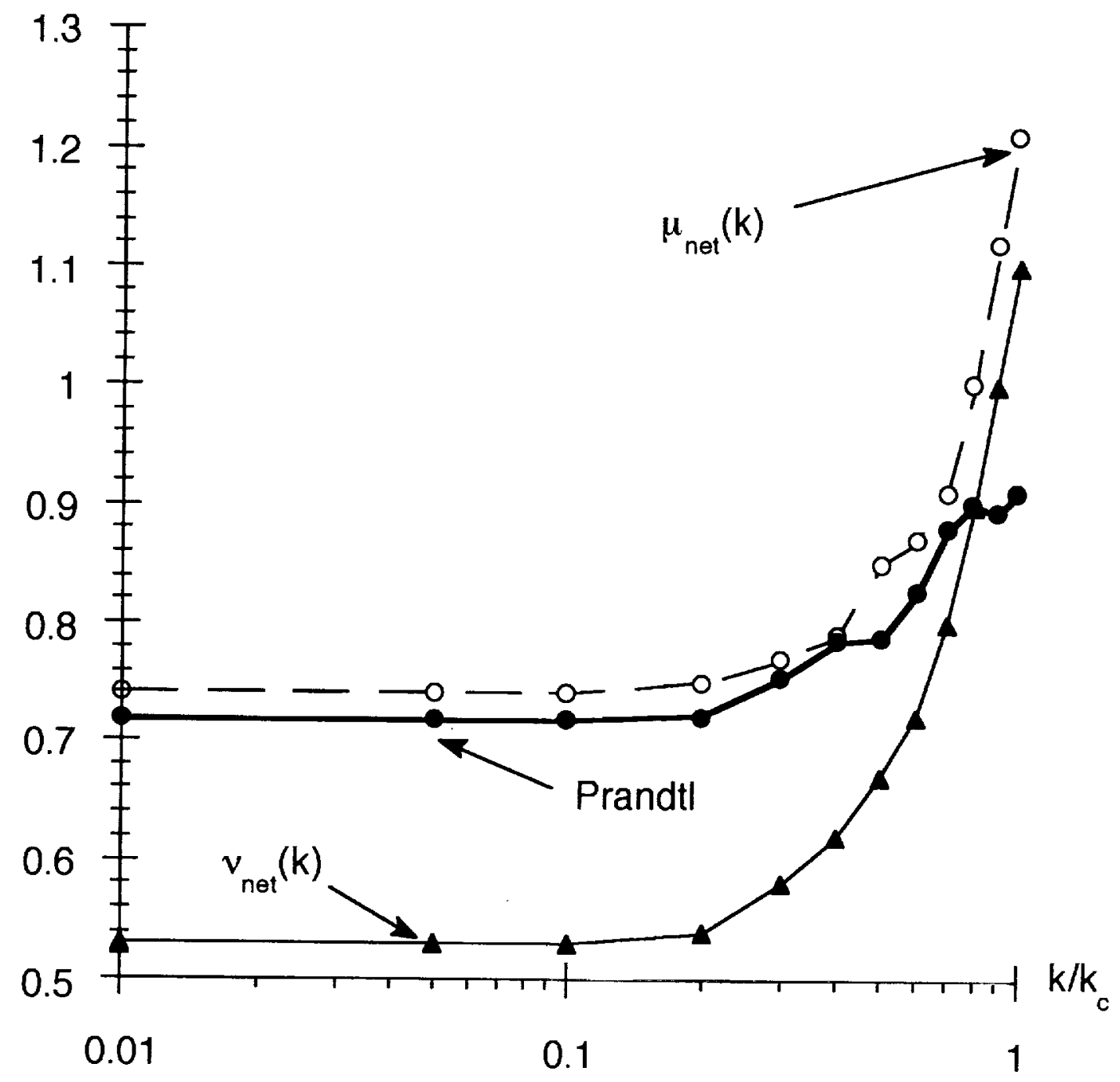

Fig. 5 The spectral eddy viscosity, diffusivity and Prandtl number in the differential subgrid shell thickness, $f \rightarrow 1$, limit. The parameter $r$ is 2 . 


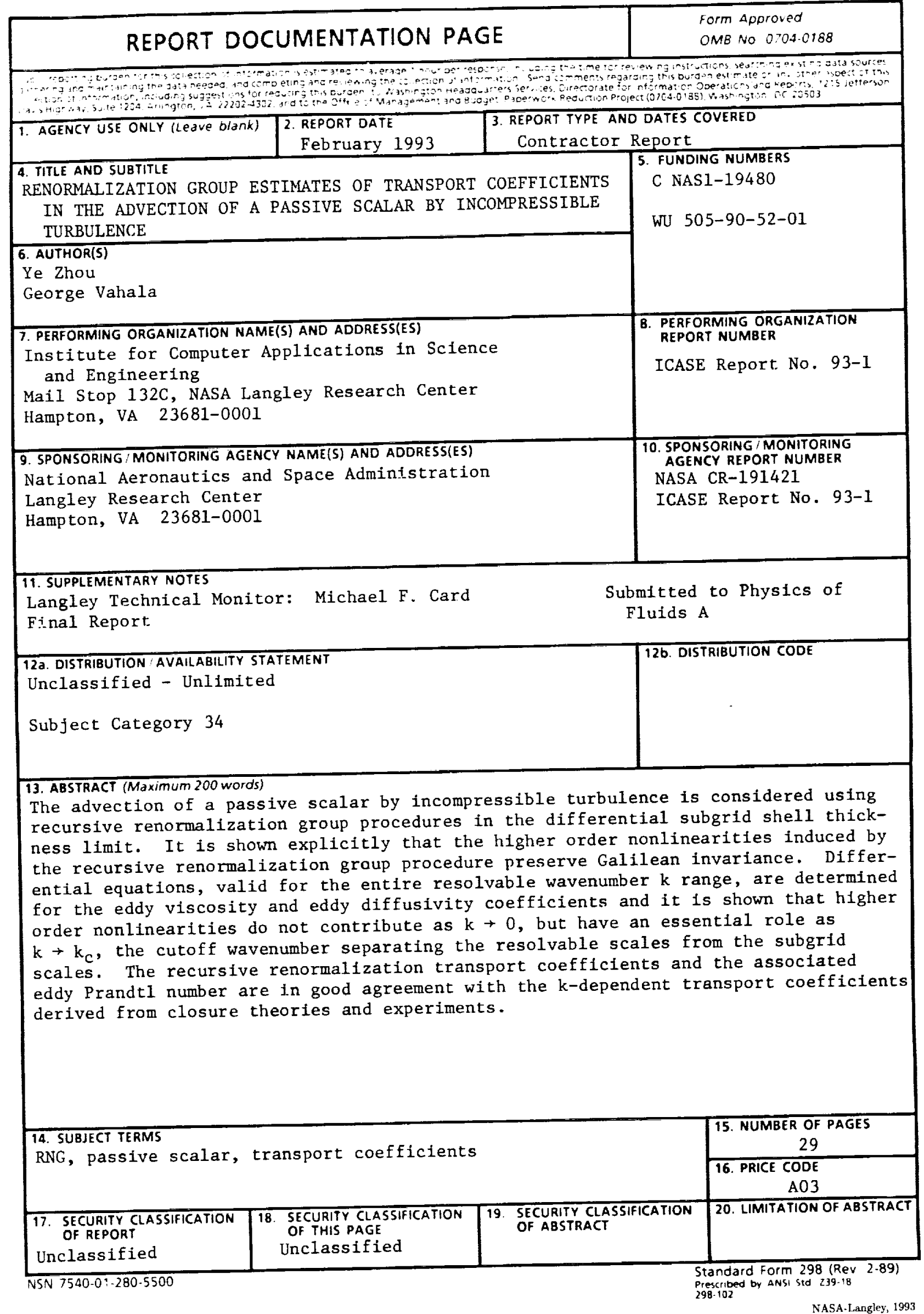

